\title{
Requirements to Establishing Confidence in Physiologically Based Pharmacokinetic (PBPK) Models and Overcoming Some of the Challenges to Meeting Them
}

\author{
Sheila Annie Peters ${ }^{1} \cdot$ Hugues Dolgos ${ }^{1}$
}

Published online: 25 June 2019

(c) The Author(s) 2019

\begin{abstract}
When scientifically well-founded, the mechanistic basis of physiologically based pharmacokinetic (PBPK) models can help reduce the uncertainty and increase confidence in extrapolations outside the studied scenarios or studied populations. However, it is not always possible to establish mechanistically credible PBPK models. Requirements to establishing confidence in PBPK models, and challenges to meeting these requirements, are presented in this article. Parameter non-identifiability is the most challenging among the barriers to establishing confidence in PBPK models. Using case examples of small molecule drugs, this article examines the use of hypothesis testing to overcome parameter non-identifiability issues, with the objective of enhancing confidence in the mechanistic basis of PBPK models and thereby improving the quality of predictions that are meant for internal decisions and regulatory submissions. When the mechanistic basis of a PBPK model cannot be established, we propose the use of simpler models or evidence-based approaches.
\end{abstract}

\section{Key Points}

To leverage the mechanistic strengths of PBPK models, it is essential to establish confidence in the mechanisms that are relevant to an application.

Establishing confidence in PBPK models is challenged by poor in vitro-in vivo correlations, knowledge gaps in system parameters and in mechanisms impacting an application, as well as parameter non-identifiability.

Uncertainty analysis and hypothesis testing can be used to overcome some of these challenges.

If the mechanistic basis of a PBPK model cannot be established, then simpler models and/or evidence-based approaches should be considered.

Electronic supplementary material The online version of this article (https://doi.org/10.1007/s40262-019-00790-0) contains supplementary material, which is available to authorized users.

Sheila Annie Peters

Sheila-annie.peters@merckgroup.com

1 Merck Healthcare KGaA, Frankfurter Str. 250, 64293 Darmstadt, Germany

\section{Introduction}

Physiologically based pharmacokinetic (PBPK) models provide a mechanistic framework in which to integrate compound and system data for prospective predictions of drug exposure in humans $[1,2]$. When scientifically wellfounded, the mechanistic basis of PBPK models can help reduce the uncertainty and increase confidence in extrapolations outside the studied scenarios or studied populations. PBPK models are therefore increasingly applied during preclinical and clinical development [1,3-7]. During preclinical drug development, PBPK can support candidate drug selection and decision making by aiding an understanding of the mechanisms driving drug exposure [8]. During clinical drug development, PBPK modelling can drive internal decisions and support regulatory submissions [9-11]. An increasing number of regulatory submissions over the last decade culminated in the recent draft guidelines by both the European Medicines Agency (EMA) [12] and the US Food and Drug Administration (FDA) [13], highlighting the growing relevance of PBPK in the pharmaceutical industry today. A recent publication from the Simcyp Consortium members [7] provided a perspective on the qualification and verification of PBPK platforms/models intended for regulatory submission. Despite the strengths of PBPK modelling approaches, most of the high-impact regulatory applications 
that resulted in labelling recommendations or study waivers have tended to be drug-drug interaction (DDI)-related [14]. Establishing confidence in PBPK models for nonDDI applications such as pediatric starting dose selection, organ impairment and absorption-related applications is challenged by the difficulty in developing mechanistically credible PBPK models or to verify and validate their prediction performance, either because drug elimination pathways cannot be well-characterized, or, when characterized, there is poor in vitro-in vivo correlation (IVIVC). This is especially true for transporter-dependent or non-cytochrome P450 (CYP)-mediated elimination pathways. The lack of a sufficient number of clinical datasets to resolve parameter non-identifiability has further limited model verification and validation. This work presents a systematic assessment of the current challenges to establishing confidence in PBPK models with respect to parameter estimation and model verification in each of the three major areas of PBPK application-absorption prediction, exposure prediction in a target population, and DDI risk assessment during drug development. These three areas cover most of the regulatory submissions. This paper also focuses on overcoming parameter non-identifiability issues through hypothesis testing, using case examples related to absorption.

\section{Impact Levels of Physiologically Based Pharmacokinetic (PBPK) Applications for Regulatory Submissions}

In a workshop on modelling and simulation hosted by the EMA and the European Federation of Pharmaceutical Industries and Associations (EFPIA), representatives from industry, academia, and regulatory agencies proposed a framework where the degree of regulatory scrutiny, level of documentation, and the need for early dialogue is proportional to the impact of the modelling activity on regulatory decision making $[15,16]$. Thus, regulatory submissions may be classified as high, medium or low impact depending on the ability of the work to replace, justify or describe an evidence base. For example, PBPK models that support regulatory filing for labelling negotiations or study waivers are considered 'high impact' applications. In general, these tend to be DDI-related. Pediatric starting dose selection or the study design may be considered an example of an application with moderate impact.

\section{Qualification of PBPK Platforms}

PBPK models are generally built in commercial platforms such as GastroPlus (http://www.simulations-plus.com), PK-Sim ${ }^{\circledR}$ (http://www.open-systems-pharmacology.org/) or Simcyp (http://www.simcyp.com) that come with their own compound and population libraries. The EMA guidelines require qualification of these platforms [12]. PBPK platform qualification is defined as a version-specific evaluation to demonstrate its reliability for one or several intended purposes. It involves ensuring proper implementation of computational functionalities, accurate mathematical representation of the physiological processes, reliable system parameters for the library of populations, model verification for the library compounds, transparency regarding the source of system and compound data as well as assumptions in the system, version controlling, quality-controlled software installation, and evaluation of the predictive performance for high-impact applications for the intended purpose using a large, independent, diverse dataset.

\section{Key Questions and Strategies for Different PBPK Applications}

Boxes 1 and 2 summarize the key questions that are addressed through PBPK modelling and simulations for some high-impact DDI applications. They also outline the modelling strategies employed to address these questions, as well as the possible outcomes that may be expected for regulatory submissions. Box 3 summarizes the key questions addressed by PBPK for moderate-impact nonDDI applications. The PBPK modelling strategy for an application depends on the impact level and the nature of the question that needs to be addressed. In general, the modelling strategy for a high-impact application involves the following:

- Model development Building a PBPK model for a new chemical entity (NCE) by integrating its physicochemical properties, measured in vitro data that are relevant to the key question to be addressed, and estimated sensitive or critical parameters from clinical pharmacokinetic (PK) data when they become available.

- Model verification An iterative process of comparing model-simulated exposure with independent clinical data (datasets that were not used in model development steps) to establish confidence in the model-simulated exposure. If model simulations do not match the clinically observed exposure within a predefined acceptance criterion, the model parameters are refined to fit the observations and then verified again. An acceptance criterion that is flexible, clinically relevant and based on sample size, parameter variance, therapeutic index and exposure-response relationship has been proposed [17].

- Model validation Following model verification, the prediction performance of a model needs to be validated 
Box 1 Key questions (Q), modelling strategies and possible outcomes for high-impact regulatory submissions: NCE as a perpetrator of DDI. DDI drug-drug interaction, $N C E$ new chemical entity, $C L_{\text {int }}$ intrinsic clearance, $C Y P$ cytochrome P450, EM extensive metabolizers, $I M$ interme- diate metabolizers, $f g$ fraction escaping intestinal loss, $f_{m} C Y P$ fraction metabolized by an isoform, FiM first in man, $S A D$ single ascending dose, $M A D$ multiple ascending dose, $P K$ pharmacokinetics, $P G$ pharmacogenomic, $P M$ poor metabolizer, $C L$ clearance, $C L / F$ apparent clearance

\section{High impact regulatory submissions: Victim DDI, genetic variations}

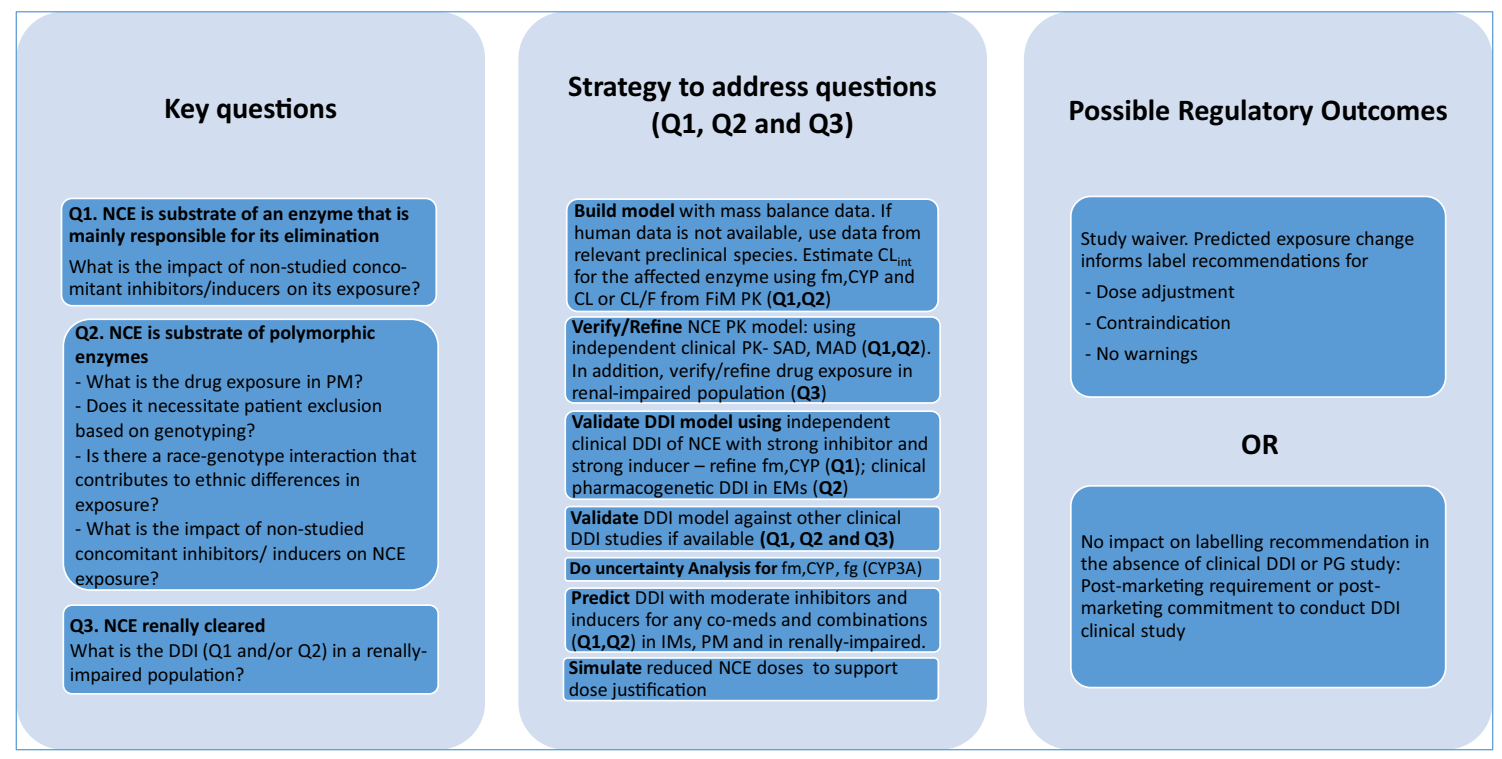

Box 2 Key questions, modelling strategies and possible outcomes for high-impact regulatory submissions: $f g$ fraction escaping intestinal loss, $K i$ reversible inhibition constant, $K I$ inhibitor concentration at half maximal inactivation, NCE as a victim of DDI. DDI drugdrug interaction, $Q x$ Quarter $x, N C E$ new chemical entity, $S A D$ single ascending dose, $M A D$ multiple ascending dose, $P K$ pharmacokinetics, $P$ - $g$ P P-glycoprotein, OATP organic anion transporting polypeptide, $O C T$ organic cation transporter, $B C R P$ breast cancer resistance protein, $E C_{50}$ half maximal effective concentration, $E_{\max }$ maximum effective concentration

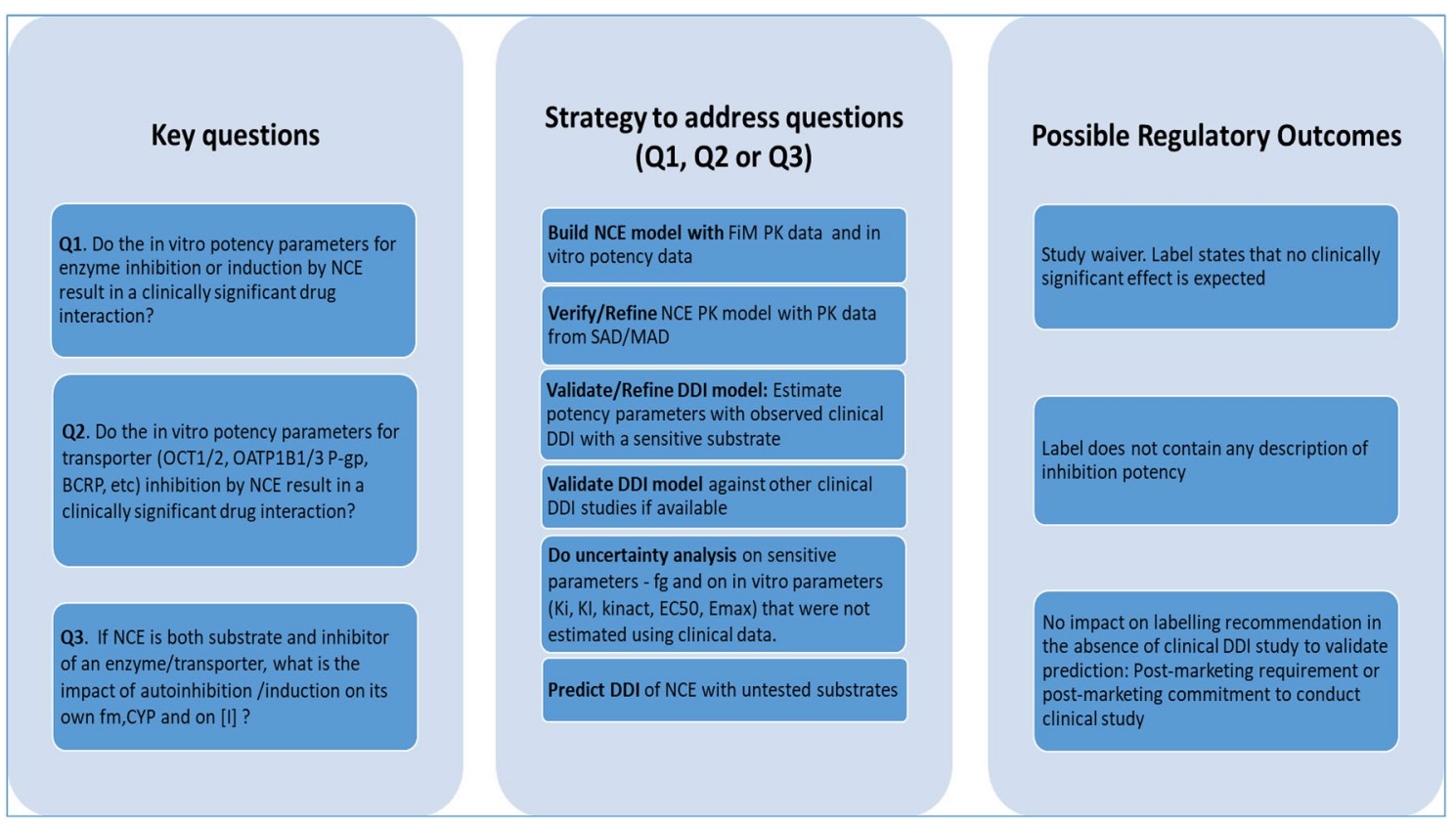

against relevant clinical data (eg, predicted within 2-fold of the observed) to demonstrate that the proposed model is 'fit for purpose', before applying the model for predict- ing an untested scenario. For example, a PBPK model of a CYP inhibitor, verified against observed PK profiles in a first-in-human $(\mathrm{FiH})$ trial may be validated for the 
Box 3 Key questions for moderate impact non-DDI regulatory submissions. $D D I$ drug-drug interaction, $P K$ pharmacokinetics, $P P I$ proton pump inhibitor, $P$ - $g p$ P-glycoprotein, $C_{\max }$ maximum concentration

\section{Key questions}

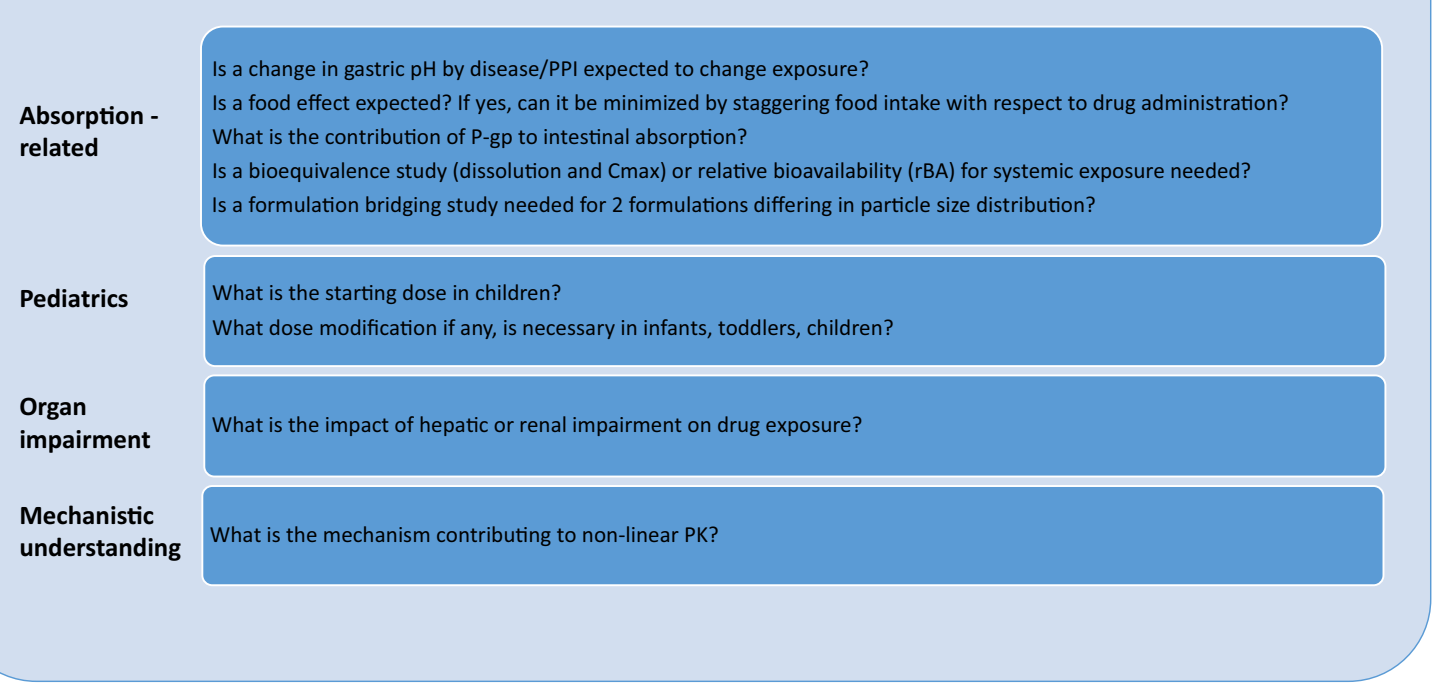

purpose of predicting drug interaction with one sensitive substrate before it is applied to prospectively predict interactions with other untested CYP substrates. Validation with one tested scenario would be enough to provide the confidence needed for the prospective predictions of multiple untested scenarios. If the model is developed in a PBPK platform that is already qualified for an intended purpose using an independent, large, diverse dataset, this validation step may be skipped.

- Sensitivity analysis identifies sensitive model parameters among the in vitro-generated input parameters for which an uncertainty analysis needs to be performed.

- Model prediction A validated model can be applied to make prospective predictions for an unstudied population, or used to simulate an unstudied scenario (see example under 'Model validation').

A description of PBPK model qualification and verification is presented elsewhere [18]. However, in this current work, we distinguish between model verification and validation. While verification is a necessary step in a modelling exercise, in which model-simulated exposure is compared with independent clinical data (datasets that were not used in the model development steps), validation refers to the evaluation of the predictive performance of the model and may be part of either platform qualification or a regulatory submission.

\section{Requirements for Establishing Confidence in the Utility of PBPK Models for the Three Broad Categories of Applications}

The unique strength of PBPK modelling and simulations lies in its mechanistic basis. To leverage this strength, it is important to establish confidence in a mechanistically credible model that is validated for a particular purpose. This calls for a good understanding of mechanisms relevant to the question being addressed. The requirements to establish confidence in the application-relevant mechanisms depends on the type of application. For the three broad areas of PBPK application (absorption and formulation-related applications, extrapolation from a base population to pediatrics or special populations, and DDI) that cover most of the regulatory submissions, these requirements are presented in Fig. 1.

Absorption and formulation-related applications of PBPK modelling include (1) prediction of oral drug absorption by integrating drug permeability, dissolution, particle size and controlled/modified-release rates and formulation selection based on the model-predicted absorption; (2) prediction of the effects of food and/or proton pump inhibitors (PPI) on drug absorption; and (3) demonstration of bioequivalence of formulations through numerical or mechanistic IVIVC to support biowaivers. In this article, gut bioavailability $\left(F_{\mathrm{g}}\right)$ is defined as the product of fraction absorbed $\left(f_{\text {abs }}\right)$ and fraction escaping intestinal loss $\left(f_{\mathrm{g}}\right)$, and is further illustrated in electronic Supplementary Figure 1. Intestinal loss $\left(1-f_{\mathrm{g}}\right)$ is the loss of a drug due to gut metabolism or transporter-mediated 

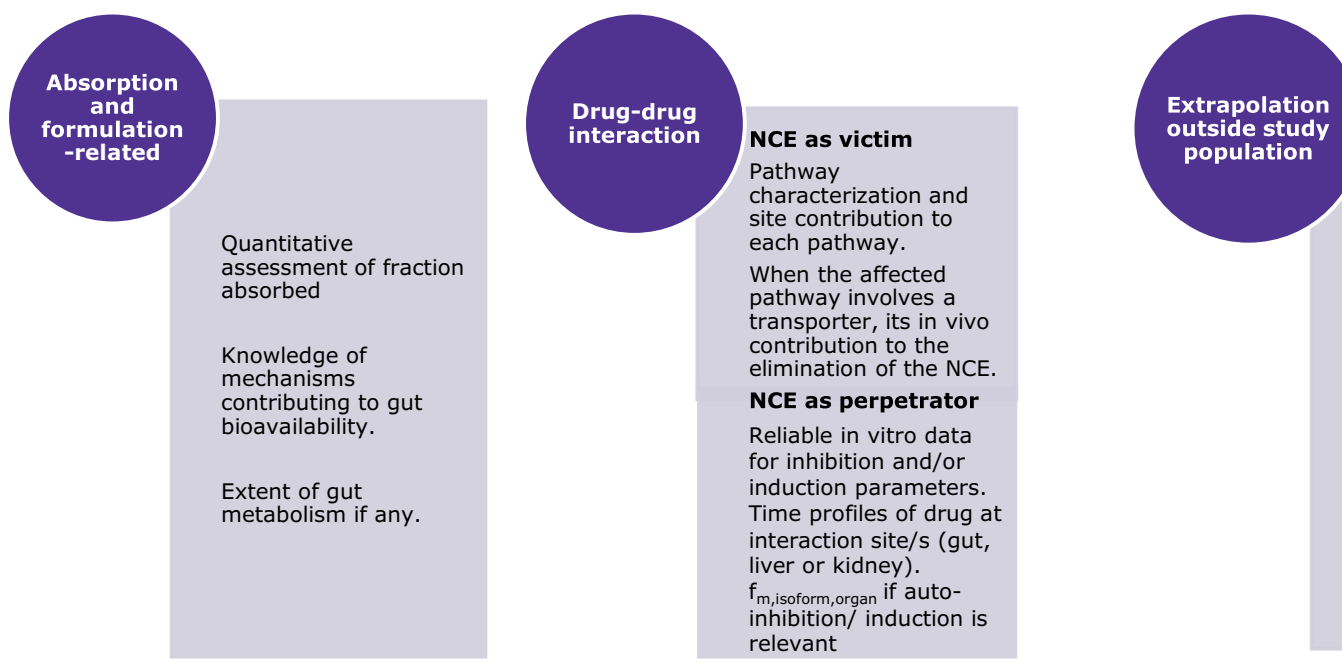

Mechanistic

understanding of PK in the base population A knowledge of how the relevant mechanisms are altered in the target population relative to the base population.

${ }^{1}$ Low variability in clinical PK in base population to allow for parameter estimation. Good in-vitro to in vivo correlation for $f_{m, \text { cyp }}$ in base population

Fig. 1 Requirements that will allow a high level of confidence in PBPK predictions for the three broad categories of applications. The placement of these three categories of applications along the value chain is also depicted. ${ }^{1}$ The greater the variability and smaller the size of the cohort, the larger the range of the estimated parameter. If

intestinal efflux. These definitions are intended to appreciate the distinction between gut bioavailability and fraction escaping gut metabolism, often used interchangeably in the literature. At doses where intestinal efflux can be considered saturated, gut metabolism is assumed to be the sole contributor to intestinal loss. Confidence in absorptionrelated predictions is expected to be high when quantitative assessment of $f_{\text {abs }}$ is reliable. For small molecule drugs that are sufficiently lipophilic (neutral or basic with log $P>1.8$ ) to allow assumption of good permeability, the in vivo relevance of in vitro solubility and dissolution play a key role in the quantitative prediction of $f_{\text {abs }}$. In addition, knowledge of mechanisms contributing to gut bioavailability other than absorption, e.g. efflux and gut metabolism should either be known to be irrelevant (e.g. NCE is not a substrate of efflux transporters or enzymes expressed in the gut) or, when relevant, should be quantitatively assessed. For example, if gut metabolism is known to be relevant for the NCE, then quantifying the metabolic contribution of the gut requires metabolite measured in intravenous and oral routes. For CYP3A substrates, reasonable quantification is possible even with in vitro data [19]. For non-CYP drivers of gut metabolism, the availability of PK data following intravenous administration is indispensable in the this range is close to the entire range of plausible values, the exercise of parameter estimation is rendered less valuable. $P K$ pharmacokinetics, $N C E$ new chemical entity, $D D I$ drug-drug interaction, $P B P K$ physiologically based pharmacokinetics

quantitative mechanistic understanding of gut bioavailability. In the absence of intravenous data, for poorly soluble compounds, it is difficult to characterize the mechanisms relevant for absorption-related applications.

Application of PBPK to extrapolate drug exposure from a base population (usually healthy, adult, Caucasian) to other populations (pregnant, obese, smokers, hepatically/renally impaired, pediatric, elderly and different ethnicities) requires a mechanistic understanding of the drug PK in the base population, as well as a knowledge of how these mechanisms are altered in the target population (population in which the prediction is desired). This requires that the metabolic and elimination pathways, as well as the site contributing to each of the metabolic pathways (Electronic Supplementary Figure 2) are well-characterized. A combination of clinical DDI and PK data can also provide fraction metabolized $\left(f_{m}\right)$. If an NCE is a transporter substrate, the in vivo contribution of the transporter to its elimination should be additionally well-understood. A good recovery of the in vivo clearance in the base population from in vitro intrinsic clearance $\left(C L_{\text {int }}\right)$ is then necessary to adjust for differences in protein levels in the target population and will ensure that unique mechanisms relevant to the target population can be accounted for. When multiple CYP enzymes are responsible 
for the metabolism, pathway contributions should be verified through drug interaction studies and human absorption, distribution, metabolism and elimination (ADME)/ mass balance studies when they become available. If only a single major CYP is involved in the metabolism in the base and target populations, $C L_{\text {int }}$ may be derived from observed clearance in an intravenous PK study in the base population. A low variability in clinical PK (approximately 30\% coefficient of variation) in the base population will allow for a robust estimation of parameters. In addition, for an oral drug whose gut bioavailability is $<1$, the contribution of absorption, efflux or gut metabolism to gut bioavailability should be well-understood, as described for absorption-related applications. This will allow for appropriate corrections in parameters by accounting for differences between the base population and target populations.

Most of the applications of PBPK modelling and simulations in regulatory submissions are related to DDI, in which an NCE is either assessed as a victim/perpetrator of enzyme (CYP and non-CYPs)/transporter inhibition or CYP induction when coadministered with other drugs. To establish confidence in the utility of PBPK modelling for assessing an NCE as a victim drug, the metabolic and elimination pathways, as well as the site contributing to each of the metabolic pathways, should be well-characterized, as described for extrapolation to the target population. If the NCE is a transporter substrate, the contribution of transporter to elimination of the NCE should be additionally well-understood. For NCE as a perpetrator, it is enough to have the model-simulated profiles of drug exposure at interaction sites (gut, liver or kidney) derived from clinical data at steady-state and reliable in vitro data for inhibition and/or induction parameters. If the perpetrator drug also relies on the affected pathway for its own metabolism, then the fraction metabolized by an affected enzyme in the organ of interest $\left(f_{\mathrm{m} \text {,isoform,organ }}\right)$ is additionally required for the affected enzyme to account for potential auto-inhibition/induction. Thus, the requirements shown in Fig. 1 may be adapted to fit the purpose of the PBPK model, depending on the mechanisms relevant for a given drug and the availability of clinical data for model building.

In general, for all three major applications of PBPK, the fewer the mechanisms impacting the drug exposure (drug dissolution, and metabolic and elimination pathways), the fewer the associated parameters, and therefore overall uncertainty, and the greater the confidence in model predictions. Thus, Biopharmaceutics Classification System (BCS) I compounds for which elimination is driven by single (or major) CYP-mediated metabolism are likely to be associated with the least parameters, least overall parameter uncertainty, and therefore the highest confidence in prospective predictions (Electronic Supplementary Figure 3), as exposure of these compounds is not likely to be limited by absorption or impacted by transporters. Therefore, the only drug parameters needed for a BCS I perpetrator drug, for example, are parameters related to drug clearance, enzyme inhibition or induction potency of the drug. However, biowaivers are granted for BCS Class I drugs even without modelling. BCS Class II-IV drugs with their solubility- and/or permeability-limited, transporter-dependent exposure are subject to absorption and DDI challenges that can be addressed with PBPK. Applications include absorption [20, 21], PPI effect [22], food effect prediction [23, 24], bioequivalence assessment through IVIVC for getting a biowaiver for formulation bridging, and DDI assessment [25-30], to name a few. A comprehensive list of applications is covered by Shebley et al. [7].

\section{Challenges to Establishing Confidence in a PBPK Model}

When mechanisms that are relevant to an application of interest can be identified, associated parameters well-established, and the PBPK model built in a qualified platform is verified and validated (if necessary) against clinical data, then confidence in the predictive performance of the model is said to be high. However, there are several barriers to establishing confidence in the mechanisms related to an application. These are presented in Fig. 2.

\subsection{Model Building}

\subsubsection{Identifying Key Mechanisms Impacting an Application}

The possibility that mechanisms relevant for the in vivo disposition of a drug can go unidentified in in vitro systems cannot be dismissed. This is reflected in the large in vitro to in vivo (IVIV) disconnect in $\mathrm{CL}_{\text {int }}$ for such drugs. If the unidentified mechanisms do not impact the outcome for the intended use of the PBPK model, the IVIV disconnect should cause no concern. For example, a PBPK model built for a CYP3A inhibitor can be used for its intended purpose of assessing the DDI risk when combined with a potential CYP3A substrate, if human PK are available, even though the contributing enzymes are not well-characterized. However, if the intended purpose of the model is to assess the risk for an NCE to be a victim of CYP inhibition, a quantitative knowledge of all elimination and metabolic pathways is needed. Top-down approaches can be helpful, if it is the major metabolic pathway. 


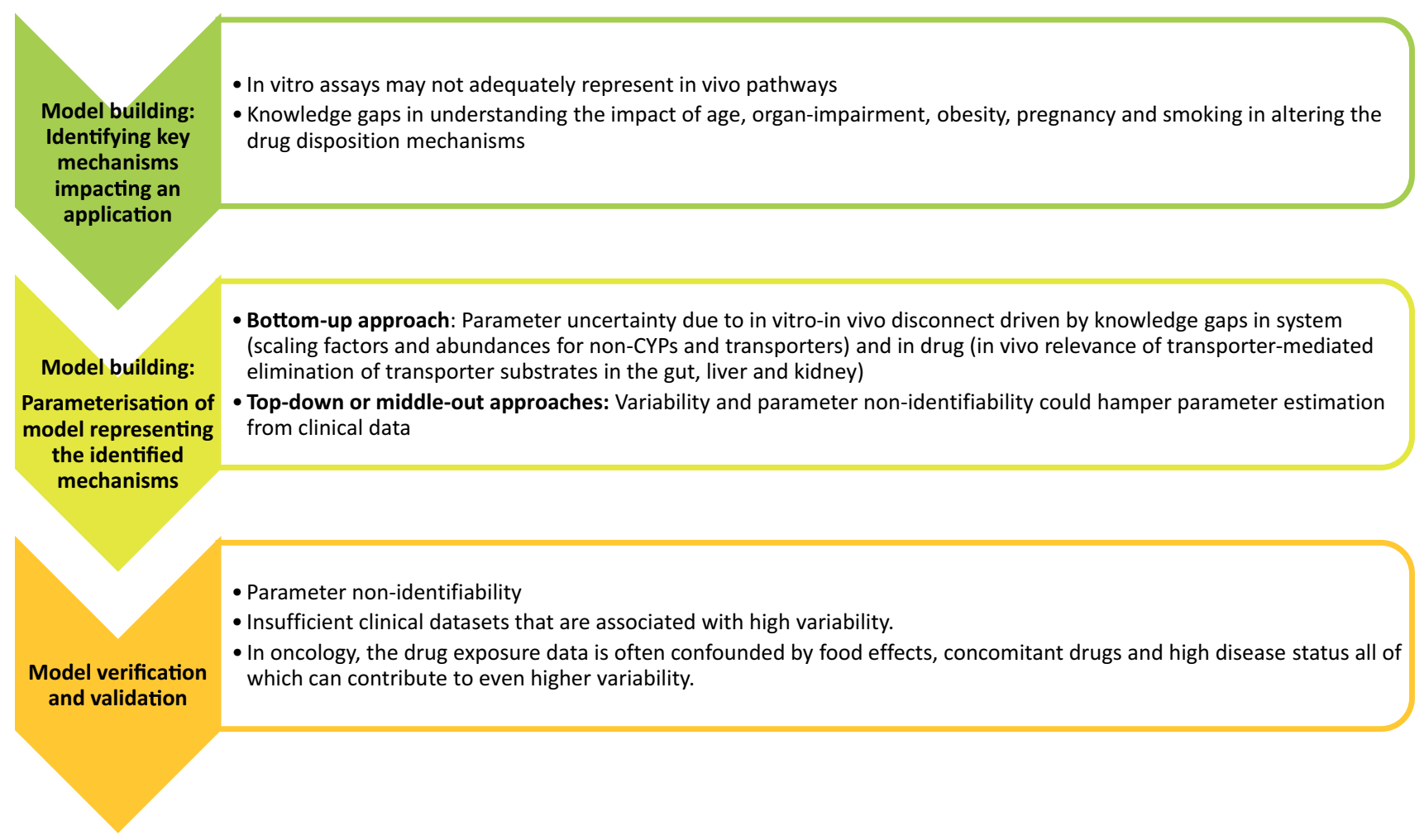

Fig. 2 Barriers to establishing confidence in the key mechanisms impacting an application. CYP cytochrome P450

\subsubsection{Model Parameterization with In Vitro Data in a Bottom-Up Approach}

PBPK model parameters needed for different categories of applications, and their typical sources, are shown in Table 1. Several experiments are carried out during drug development to identify and understand the mechanisms contributing to drug exposure. For those mechanisms that are identified, the in vivo relevance of the in vitro data generated cannot always be guaranteed. This is especially true for substrates of non-CYP enzymes and transporters for which adequate or reliable systems data, such as absolute abundances of relevant enzymes in the gut, liver and kidney and scaling factors to convert in vitro data to in vivo relevant data, are lacking. These challenges also apply for transporter substrates. A lack of understanding on the in vivo relevance of enzyme-transporter interplay, as well as a lack of quantitative knowledge on the extent of in vivo contribution of the transporter to the elimination of the drug, pose additional challenges for transporter substrates. These limitations make transporter and nonCYP-mediated DDI more difficult to predict [1]. However, there are several examples demonstrating the predictive value of PBPK for organic anion transporting polypeptide (OATP) substrates [31-35].
The implication of IVIV disconnect is that model parameters may be associated with uncertainty and may not be quantitative enough for a prospective prediction via a bottom-up approach. For example, to support the assessment of an NCE as a victim of DDI, several parameters, such as fm, fm, CYP, $\mathrm{CL}_{\text {int }}$, etc., would need to be generated from in vitro assays to characterize the metabolic and elimination pathways. This requirement is further complicated when multiple interaction sites (liver, intestine, kidney, etc.) are involved as it is challenging to assess the in vivo contribution of each organ. For a drug with multiple elimination pathways in multiple sites, mass balance studies in humans using radiolabelled compounds can identify and provide quantitative information on the routes of excretion [36], and, with additional analyses, metabolic pathways [37]. These studies aid a complete understanding of clearance and potential contributors to intersubject variability and DDIs, all of which are crucial for evaluating an NCE as a victim of drug interaction. However, it should be noted that mass balance cannot distinguish between enzyme isoforms that lead to the same metabolite. 


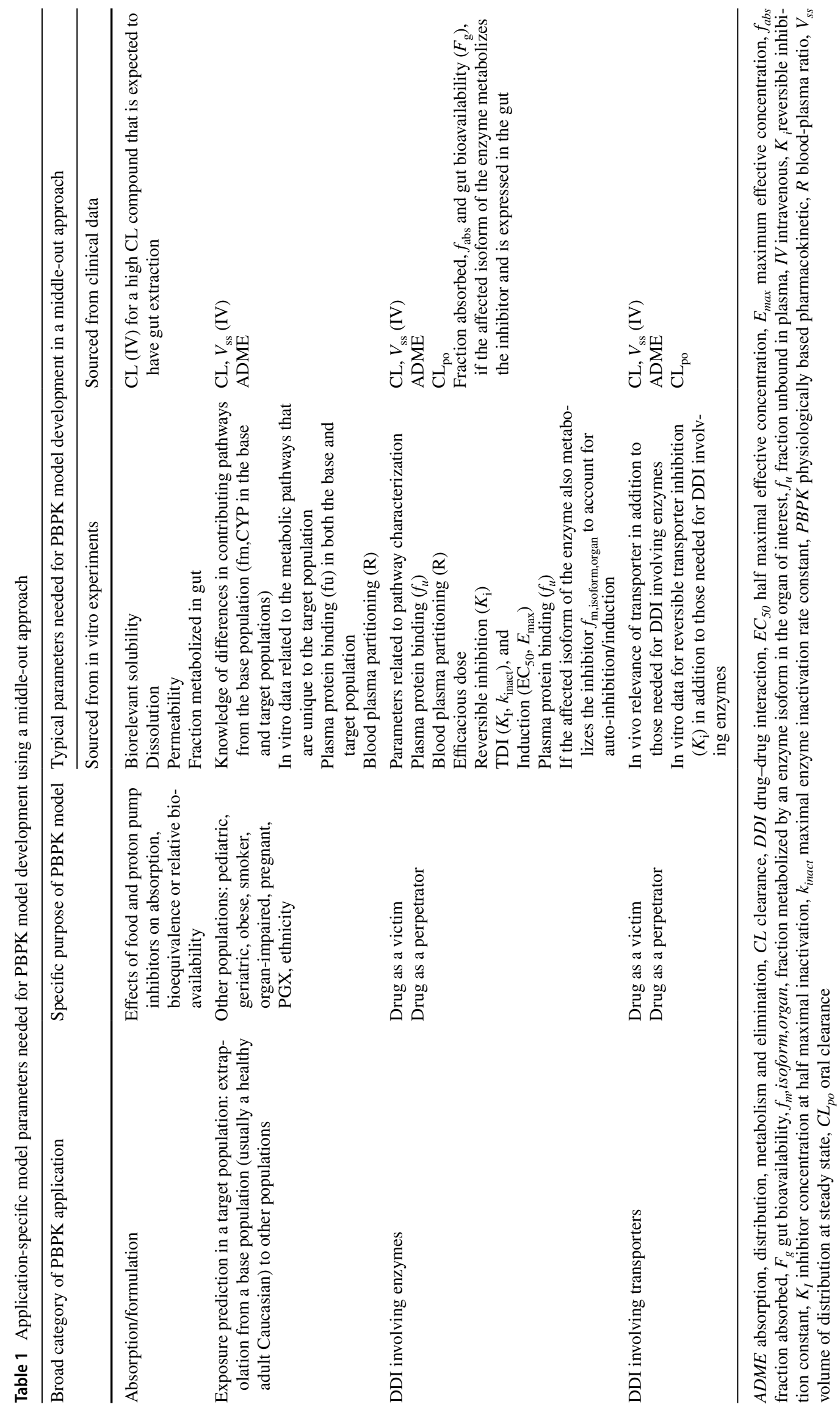




\subsubsection{Parameter Estimation from Clinical Data in Top-Down or Middle-Out Approaches}

To overcome the uncertainty associated with clearance derived from in vitro systems, a middle-out approach to model building is adopted $[18,38]$ in which clearance is obtained through parameter estimation from clinical data. This works best for a drug that is not a transporter substrate when its elimination is dominated by a single pathway. When clinical data are associated with high interindividual variability, it is reflected in the wide range of the estimated parameter. Cubitt et al. have shown that $\mathrm{CL}_{\text {int }}$ can be back-calculated from clinical data using the Simcyp simulator, even for compounds that show high interindividual variability in clearance [39]. These authors calculated a confidence interval from geometric mean and geometric standard deviation, making it possible to limit the range, by eliminating any bias from extreme individuals. In cancer patients, high variability in PK profiles usually from a small cohort renders estimated parameters less reliable as the true mean cannot be captured.

\subsubsection{Parameter Non-Identifiability as a Barrier to Deconvolute Mechanisms Contributing to Gut Bioavailability}

Gut bioavailability of an orally administered drug is determined by solubility, permeability, gut metabolism and efflux. Gut bioavailability of a BCS Class I, low clearance NCE that is not a substrate of P-glycoprotein (P-gp), breast cancer resistance protein (BCRP), CYP3A, or other drugmetabolizing enzymes, expressed in the gut is expected to be 1 . For other drugs, gut bioavailability can be $<1$ due to solubility-limited absorption or gut metabolism/efflux. The parameters related to these mechanisms cannot be distinguished using the observed plasma exposure data following oral administration of the drug as it allows only the estimation of a composite parameter comprising all parameters related to the contributing mechanisms. Thus, the mechanisms contributing to gut bioavailability (absorption, gut metabolism and efflux) are said to be non-identifiable since several sets of parameter values can result in equally good fit to the observed plasma exposure data. An intravenous ${ }^{14} \mathrm{C}$ microtracer dose of the NCE administered concurrently with an oral therapeutic non-labelled dose can be used to generate clearance and absolute bioavailability data without having to develop a conventional intravenous formulation and an intravenous toxicity and safety package [36]. In the absence of any gut metabolism and efflux, such a study, if available, can help identify solubility-limited absorption. If gut metabolism and efflux cannot be excluded (e.g. the NCE is a CYP3A and/or P-gp substrate), microtracer studies are inconclusive because plasma exposure limited by these mechanisms is indistinguishable from solubility limitation.

\subsection{Model Verification and Validation}

Ideally, a drug model built with known PK mechanisms (disposition, metabolism, elimination, transporter-mediated uptake or efflux, etc.), and using estimated or measured parameters, must be verified and validated before applying the model for prospective predictions. Table 2 shows the datasets needed for building, refining/verifying and validating drug models for various applications. Model verification and validation steps ensure the robustness of the model with well-supported assumptions, especially when confidence in the underlying mechanisms is high. Model validation is sometimes challenged by parameter non-identifiability issues that cannot be resolved due to an insufficient number of independent clinical datasets. For example, to validate a PBPK model of a CYP3A and P-gp substrate, that is intended for the prediction of food effect, it is important to quantify the relative contributions of absorption, intestinal efflux, intestinal metabolism and hepatic metabolism in order to understand the driver of food effect. However, as discussed earlier, given that the only clinical data that are commonly available are systemic concentrations following oral administration, the model parameters related to absorption, intestinal efflux, and intestinal and hepatic metabolism are said to be non-identifiable. Additional clinical studies designed to characterize absorption efflux and gut metabolism are necessary for complete resolution. In this scenario, even though the PBPK model may recover the observed clinical exposure and is therefore deemed to be verified, parameter non-identifiability is a barrier to validating the model for its intended purpose of predicting food effect. The accuracy of the prediction of food effect relies on the deconvolution of mechanisms contributing to gut bioavailability. If confidence in the relative contributions of efflux, gut metabolism and absorption to gut bioavailability is low, then food effect predictions are likely to be poor.

\section{Resolving the Challenges to Establishing Confidence in Key Mechanisms Impacting an Application}

To cover for uncertainty in measured parameters, a sensitivity analysis on model parameters is first performed to identify the most sensitive parameters on which to conduct an uncertainty analysis. The impact of uncertainty in sensitive model parameters that cannot be precisely measured on endpoints of interest is assessed by varying the sensitive parameters over a range of plausible values for compoundrelated parameters, and over the 5th to 95th percentile of 
Table 2 Clinical data sources for PBPK model development, verification and validation

\begin{tabular}{|c|c|c|}
\hline Application & $\begin{array}{l}\text { Clinical data used for model development, } \\
\text { parameter estimation, verification }\end{array}$ & Data for model validation \\
\hline $\begin{array}{l}\text { Absorption: modified release formulation } \\
\text { development }\end{array}$ & $\begin{array}{l}\text { Model built with IR human PK in the fed and } \\
\text { fasted states }\end{array}$ & Model simulations of CR validated in monkey \\
\hline Exposure prediction in a target population & $\begin{array}{l}\text { PK of the base population from a SAD and a } \\
\text { MAD } \\
\text { ADME mass balance, fm, fm,CYP } \\
\text { fu in both the base and target population }\end{array}$ & $\begin{array}{l}\text { Target population is qualified } \\
\text { Pathway validation from DDI studies in the } \\
\text { base population }\end{array}$ \\
\hline PK prediction of an untested dose/regimen & $\begin{array}{l}\text { Single dose PK from a SAD } \\
\text { Repeated dose PK from a MAD }\end{array}$ & \\
\hline $\begin{array}{l}\text { DDI: NCE is a victim drug coadministered } \\
\text { with a weak/moderate inhibitor }\end{array}$ & $\begin{array}{l}\text { PK of the victim drug from a SAD and a } \\
\text { MAD } \\
\text { ADME mass balance } \\
\text { fm, fm,CYP }\end{array}$ & $\begin{array}{l}\text { PK of the victim drug coadministered with and } \\
\text { without a strong inhibitor }\end{array}$ \\
\hline $\begin{array}{l}\text { DDI: NCE is a perpetrator of an enzyme iso- } \\
\text { form that is not involved in its metabolism }\end{array}$ & $\begin{array}{l}\text { PK of a perpetrator drug from a SAD and } \\
\text { MAD } \\
\text { In vitro } \mathrm{Ki}\end{array}$ & $\begin{array}{l}\text { Model able to recover an observed interaction } \\
\text { of NCE with a sensitive substrate }\end{array}$ \\
\hline $\begin{array}{l}\text { DDI: NCE is a perpetrator of an enzyme iso- } \\
\text { form that is involved in its own metabolism }\end{array}$ & $\begin{array}{l}\mathrm{PK} \text { of a perpetrator drug from } \mathrm{SAD} \\
\text { In vitro Ki } \\
\text { fm,CYP of inhibited isoform }\end{array}$ & $\begin{array}{l}\text { Model able to recover an observed interaction } \\
\text { of NCE with a sensitive substrate }\end{array}$ \\
\hline
\end{tabular}

$A D M E$ absorption, distribution, metabolism and elimination, $C R$ controlled release, $D D I$ drug-drug interaction, $f m$ fraction metabolized, $f m, C Y P$ fraction metabolized by CYP isoform, $f u$ fraction unbound in plasma, $I R$ immediate release, $K i$ inhibition constant, $M A D$ multiple ascending dose, NCE new chemical entity, $P K$ pharmacokinetics, $S A D$ single ascending dose

distributions for system parameters [40], based on what is known about the mechanism, rather than being arbitrary.

Parameter non-identifiability presents the greatest challenge for a proper characterization of underlying mechanisms. One way to overcome non-identifiability is to measure one or more of the non-identifiable parameters that can be reliably measured and combine with the composite parameter estimated from clinical data, to obtain the other non-identifiable parameter. Depending on the number of non-identifiable parameters in the composite parameter, the number that can be reliably measured, and the availability of relevant clinical data, complete deconvolution of the composite parameters may not be possible.

For applications that do require complete deconvolution (as in the example of food effect prediction in the previous section), hypothesis generation/testing with PBPK [8, $41,42]$ can help deconvolute the relative contributions of intestinal loss factors. This begins with identifying $\mathrm{CL}_{\mathrm{int}}$ and the tissue partition coefficient $\left(K_{\mathrm{p}}\right)$ factor (a multiplicative factor applied to all $K_{\mathrm{p}}$ in the PBPK model) that best fits the observed intravenous profile (Fig. 3). Assuming linear PK, as well as the constancy of hepatic clearance across different routes of administration, the best-fit parameters from the intravenous profile are fixed for oral PK simulations. Applying a bottom-up PBPK model, PK mechanisms underlying the observed oral PK profile can be identified using signature discrepancies of the simulated profile from the observed, as shown in Box 4 [2]. Signature discrepancies from observed data may be due to higher in vivo solubility than measured in vitro, drug-induced gastric empting delay, gut metabolism, enterohepatic recirculation, auto-inhibition/saturation of drug-metabolizing enzymes, auto-inhibition/saturation of uptake transporters and auto-inhibition/saturation of efflux transporters. The hypotheses generated by this approach may then be tested by suitable experimentation.

Hypothesis generation with PBPK can already be applied to preclinical intravenous and oral $\mathrm{PK}$ profiles to gain insight into the mechanisms underlying PK profiles in the preclinical species. When human intravenous and oral PK data become available, a similar systematic line-shape analysis with PBPK can check for the relevance of the mechanisms identified in preclinical species to humans. Two examples of hypotheses generation/testing with PBPK are illustrated in Boxes 5 and 6. Box 5 describes how hypothesis generation with PBPK helped distinguish gut metabolism from poor solubility for an NCE with gut bioavailability $<1$. In this example, a compound that was believed to have solubility-limited absorption was shown to have no solubility limitation in vivo. The poor oral bioavailability of the drug could be attributed to gut metabolism. If clinical intravenous data are available, a quantitative in vivo gut bioavailability can then be estimated and employed in DDI assessment. In the absence of clinical intravenous data, dose proportional or supra-dose-proportional exposure observed in single ascending dose (SAD) and multiple ascending dose (MAD) FiH studies can support the hypotheses generated (solubility does not limit absorption) in preclinical species. This deconvolution of PK mechanisms has the potential to 
IV

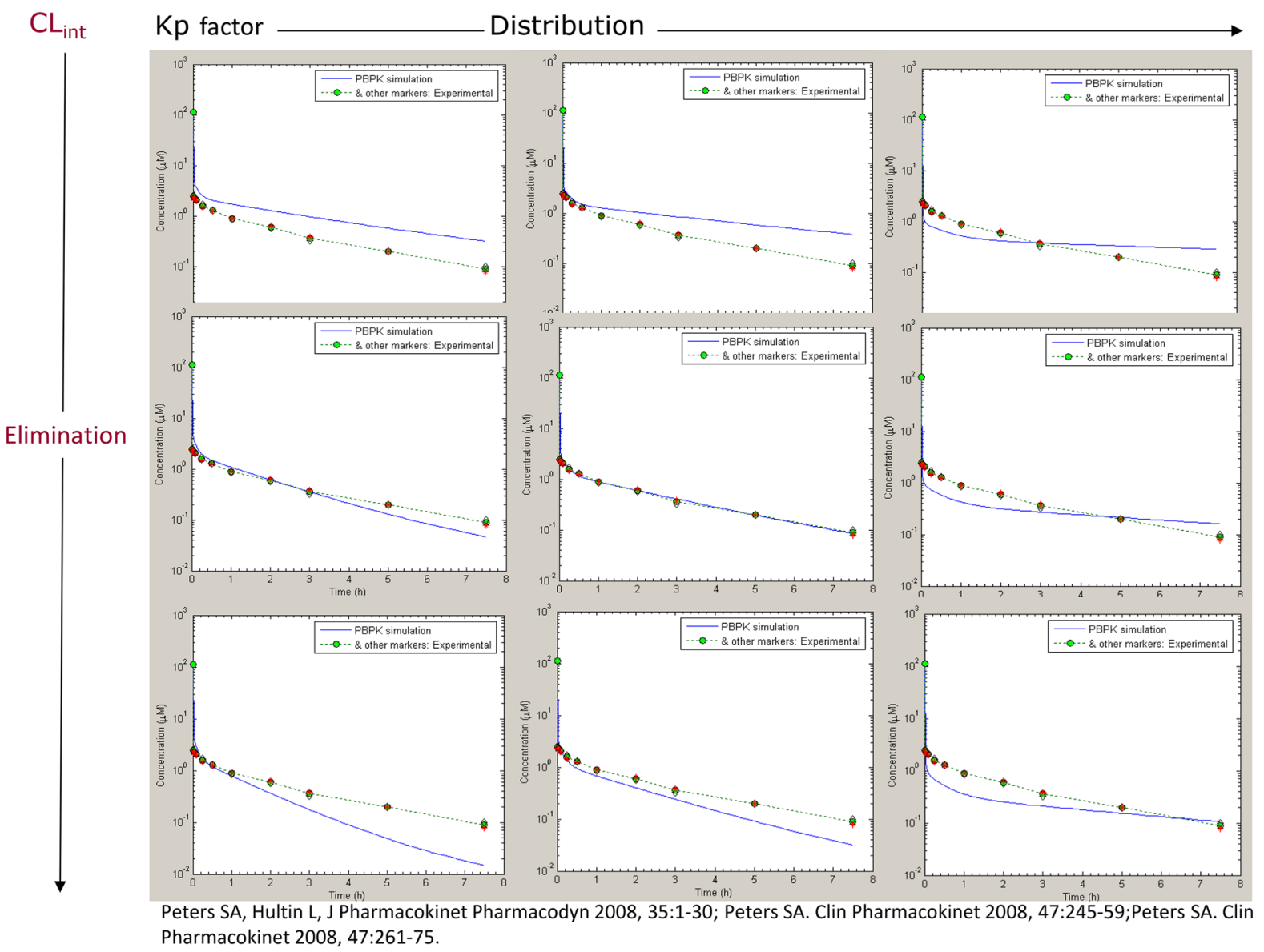

Fig. 3 Impact of changing of $\mathrm{CL}_{\text {int }}$ and multiplicative factor for $K_{\mathrm{p}}$ factors on the intravenous $\mathrm{PK}$ profile. As $\mathrm{CL}_{\text {int }}$ is increased, the profile shifts down, with the shape remaining intact. The effect of

avert any unnecessary and costly formulation development to improve oral bioavailability. Box 6 describes the use of hypothesis generation for identifying gut metabolism [42] as the principal mechanism contributing to intestinal loss. The use of in vitro solubility and permeability, with the best-fit intravenous parameters, could simulate the maximum concentration $\left(C_{\max }\right)$, but not the AUC (Boxes 6c, d). Resolving parameter non-identifiability through hypothesis generation, testing and verification can ensure a high quality of prospective predictions for absorption-related applications.

In summary, regulatory submissions demand a rigorous application of the build-verify/refine-validate-predict/ simulate modelling strategy and use of qualified platforms to address the key questions in the three broad categories of PBPK applications. In addition to building confidence in prospective predictions of PBPK models through the predictlearn-confirm process [43], understanding the mechanisms relevant to the question being addressed is highly desirable to leverage the unique strengths and mechanistic basis of PBPK models. However, there are barriers to establishing increasing the $K_{\mathrm{p}}$ factor is to change the shape of the profile. $C L_{\text {int }}$ intrinsic clearance, $K_{p}$ tissue partition coefficient, $P K$ pharmacokinetic, $I V$ intravenous, $P B P K$ physiologically based pharmacokinetics

confidence in the mechanisms relevant to an application for the building and validation of models. Hypothesis generation/testing with PBPK models can provide useful insights into mechanisms underlying observed concentration-time profiles and pave the way for improved confidence in PBPK model predictions.

\section{PBPK or Simpler Models?}

"Everything should be made as simple as possible, but not simpler" is a quote attributed to Einstein, which succinctly expresses the principle of Occam's razor. The rationale behind this generic principle is that the number of assumptions generally tends to increase with the increasing complexity of models/hypotheses, and, at some point, a complex model could become too distant to whatever is being modelled. PBPK models or simpler? The choice should depend on the value addition brought on by PBPK over 
Box 4 Signature discrepancies of predicted oral PK profiles from observed, using the PK parameters (clearance, volume of distribution and enterohepatic recirculation rate) that best fit the intravenous profile. Best fit to oral profiles were obtained by altering parameters that uniquely identify a mechanism (reference 2). $P K$ pharmacokinetics, $A U C$ area under the curve, $B C S$ Biopharmaceutics Classification System, $D D I$ drug-drug interaction, $I V$ intravenous

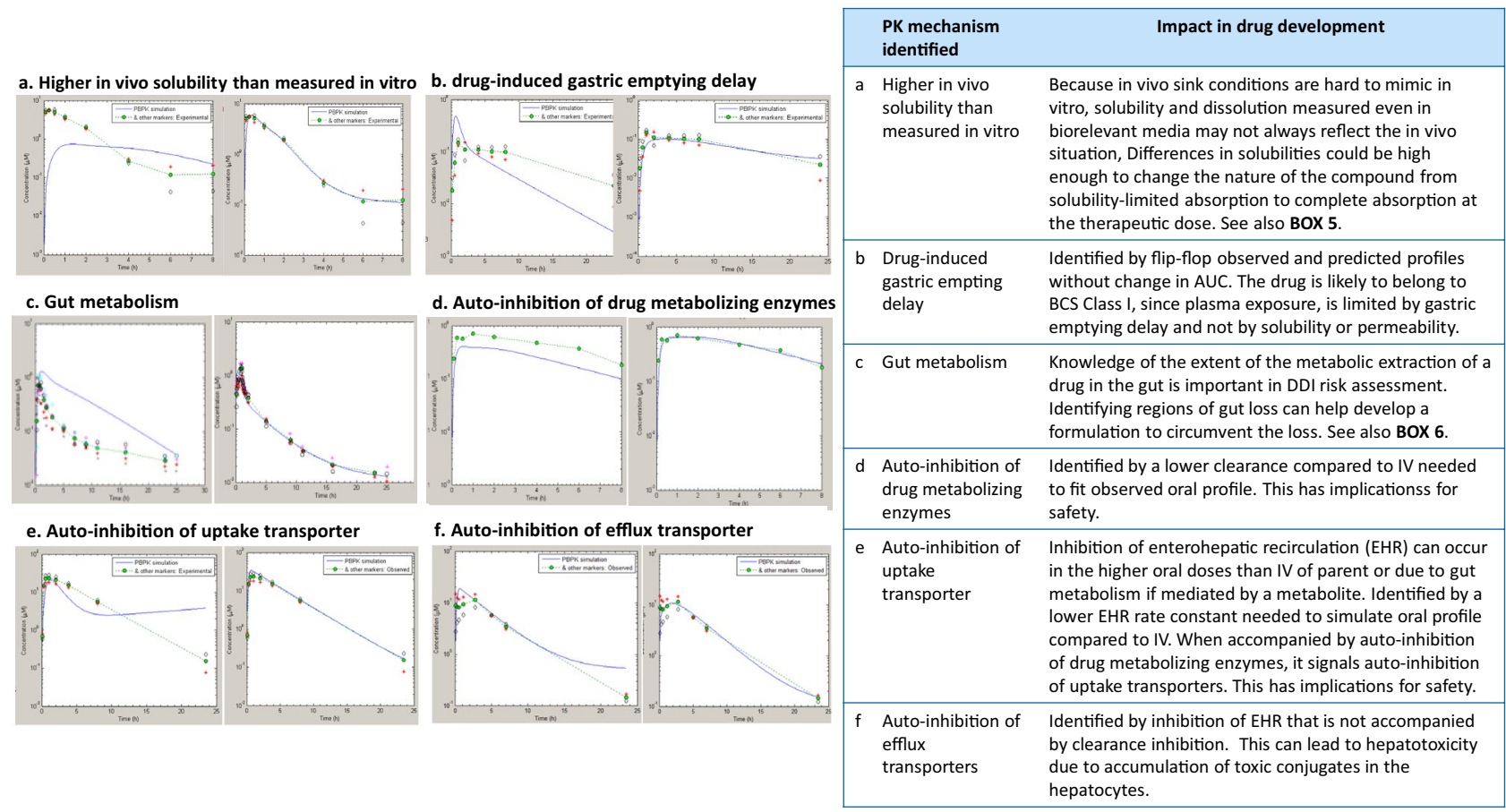

Box 5 Resolving parameter non-identifiability through hypothesis testing with PBPK simulations: identifying solubility-limited absorption (reference 2). $P B P K$ physiologically based pharmacokinetics, $N C E$ new chemical entity, $S A D$ single ascenting dose, $M A D$ multiple ascending dose, FASSIF fasted simulated small intestinal fluid, IV intravenous, $P K$ pharmacokinetics, $A U C$ area under the curve, $C L_{\text {int }}$ intrinsic clearance, $C Y P$ cytochrome $\mathrm{P} 450, K_{p}$ tissue partition coefficient
BACKGROUND

- NCE is a weak base with poor solubility and dissolution.

- Can this explain its low bioavailability in rat?

- Is there a need for a special / enabling formulation to improve its exposure?

- Is a food and/or ARA effect expected?

DATA NEEDED FOR INITIAL MODEL BUILDING Physicochemical properties logP, pKa, fu, R, particle size

In vitro

FASSIF solubility, dissolution, permeability PRECLINICAL DATA THAT AID HYPOTHESIS GENERATION Supra dose proportional exposure in toxicology studies in pre-clinical species (e.g., rat) over a very wide dose-range. HYPOTHESIS

Perhaps solubility does not limit absorption HYPOTHESIS TESTING IN PRECLINICAL SPECIES PBPK simulations can help in testing the hypothesis if solubility is really limiting absorption.

In the example shown in the Figure, the observed profile coud be simulated with a much higher solubility, but not a higher permeability.

The in vivo solubility is at least 20 times highe VERIFICATION IN HUMAN AND CONCLUSIONS Dose proportional or supra dose-proportional exposure and short tmax observed in SAD and MAD?

If IV is available: Does the in vivo solubility provide a better fit to observed oral profile with IV best fit PK parameters? ARA effect with omeprazole (a drug inducing gastroparesis) shows flip-flop kinetics with no change in AUC? If the answers to any one is YES, and if hypothetical increase in solubility does not alter simulated exposure, solubility may not limit absorption, no enabling formulation is necessary, no food or ARA effect is expected and PBPK model can be used to show equivalence of 2 formulations

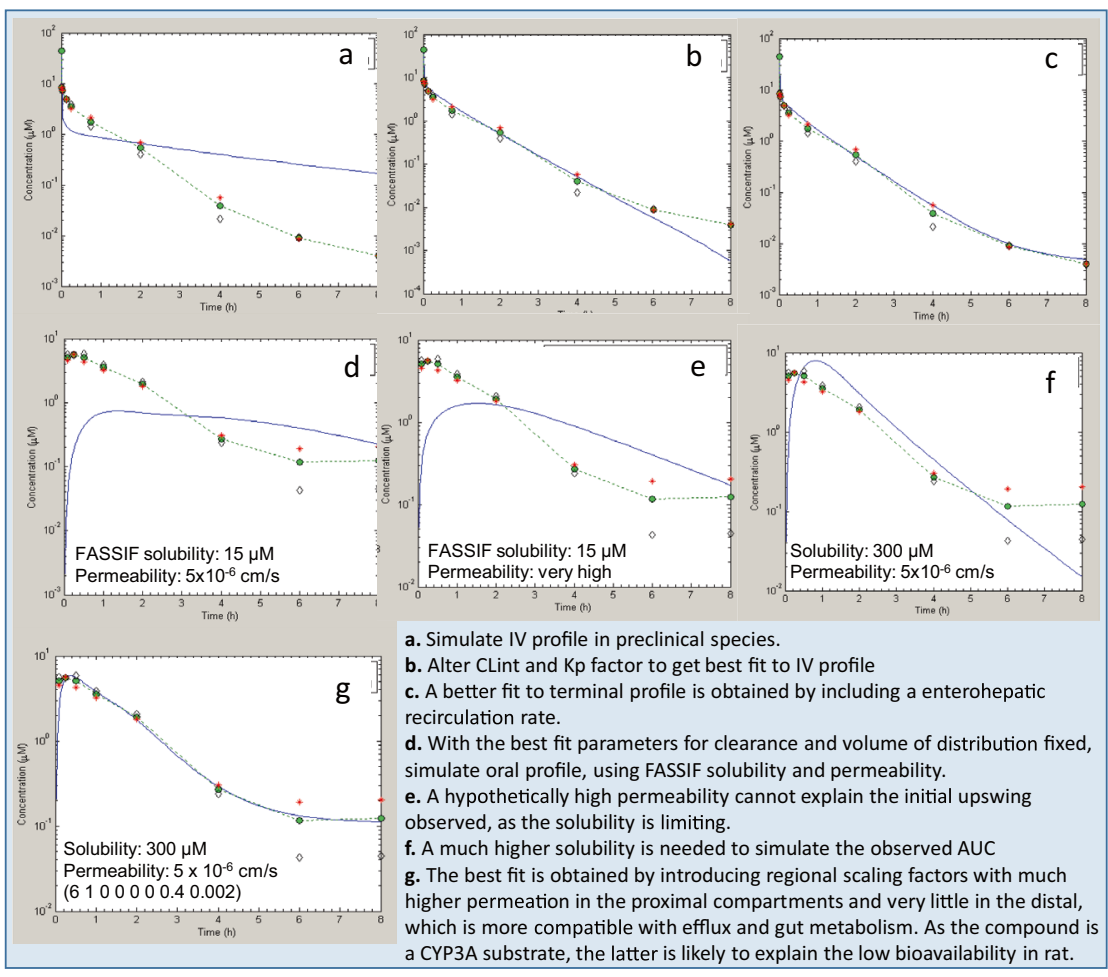


Box 6 Resolving parameter non-identifiability through hypothesis testing with PBPK simulations: identifying gut metabolism (reference 43). $P B P K$ physiologically based pharmacokinetics, $N C E$ new chemical entity, $C Y P$ cytochrome $\mathrm{P} 450, I V$ intravenous, $P K$ pharmacokinet- ics, $S A D$ single ascending dose, $M A D$ multiple ascending dose, $D D I$ drug-drug inhibition, $F_{g}$ gut bioavailability, $C L_{i n t}$ intrinsic clearance, $P$-gp P-glycoprotein, $K_{p}$ tissue partition coefficient
BACKGROUND

- NCE is a substrate of CYP3A.

- Is the compound metabolized in the gut? If so, to what extent?

DATA NEEDED FOR INITIAL MODEL BUILDING

Physicochemical properties

$\log$, pKa, fu, R,

In vitro

FASSIF solubility, dissolution, permeability

PRECLINICAL DATA THAT AID HYPOTHESIS GENERATION Oral bioavailability in rat is inconsistent with what is expected from IV clearance.

Supra dose-proportional exposure in toxicology studies in rat over a very wide dose-range

HYPOTHESIS

Gut metabolism driven intestinal loss

HYPOTHESIS TESTING IN PRECLINICAL SPECIES

Hypothesis testing with rat PK profiles identifies an intestinal loss not driven by solubility limitation of absorption but possibly due to gut metabolism.

VERIFICATION IN HUMAN AND CONCLUSIONS Dose proportional or supra dose-proportional exposure observed in SAD and MAD?

If IV is available:

If one major metabolite is produced in the gut, a higher

metabolite to parent ratio following oral administration compared to IV (Figure above)

In the absence of solubility limitation, an in vivo $\mathrm{Fg}$ can be

derived (Figure below) and employed in DDI assessment.

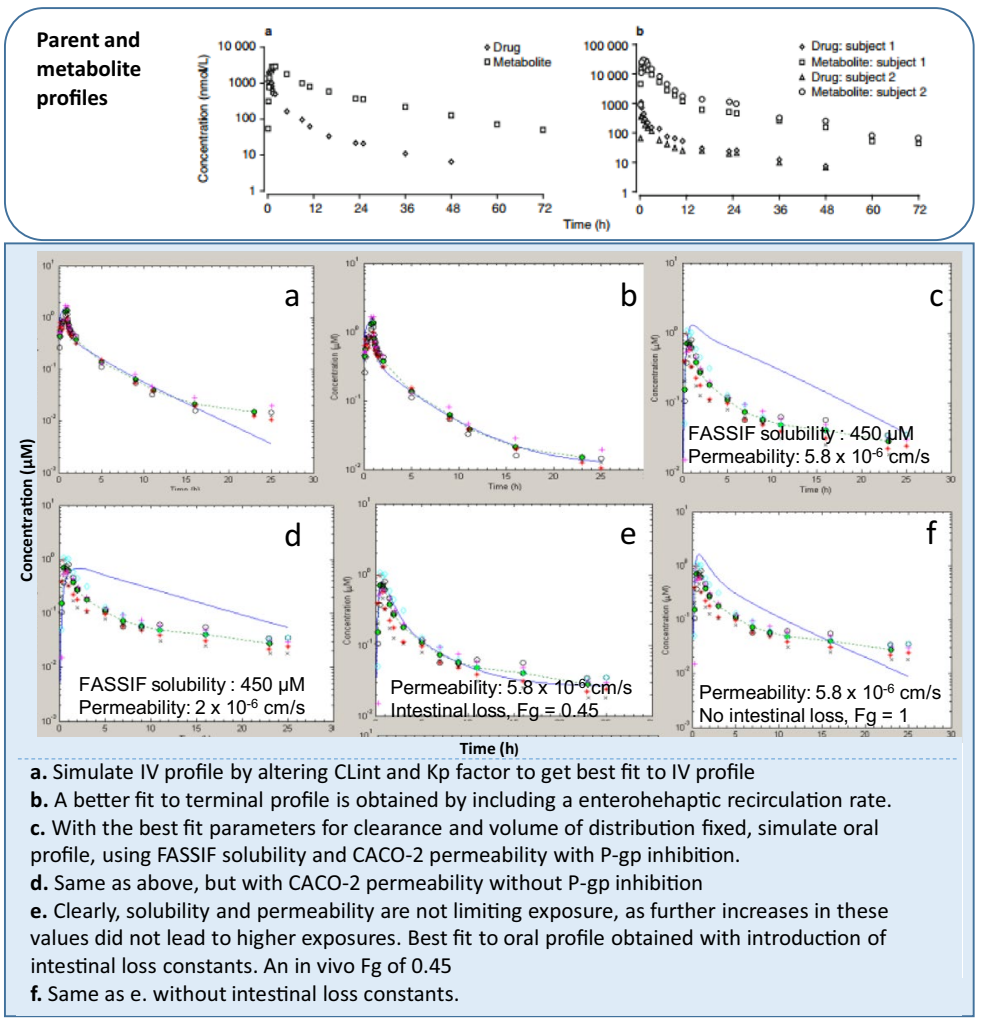

simpler methods for a particular drug in a particular application, given the challenges.

The steps involved in establishing confidence in PBPK models, and situations in which simpler models may be considered, are summarized in Fig. 4. To begin with, a fit-for-purpose PBPK model is built by identifying the key mechanisms that are relevant to the purpose of the application. Next, sensitive parameters pertinent to the identified mechanism should be estimated/derived from clinical data if possible. If the PK profile simulated by the model can be verified against an independent clinical dataset satisfying the acceptance criterion, then provided the requirements for establishing confidence in the model are met (Fig. 1), the model can be validated for its purpose before applying it for prospective prediction to an untested scenario. In the absence of satisfactory validation, it is possible that confidence in the key mechanism needs to be established, perhaps through hypothesis generation and testing.

If the verification criterion is not satisfied, further model refinement may be necessary through parameter changes that uniquely improve the fit to the clinical data. If multiple parameter changes lead to the same outcome, the nonidentifiability should be resolved through hypothesis generation and testing. If an hypothesis cannot be verified, then the mechanistic strengths of PBPK cannot be leveraged, and simpler methods supported by a totality of evidence approach should be preferred.

To illustrate the strategy laid out in Fig. 4, consider the example of a CYP3A-metabolized, poorly soluble, weakbase NCE for which a bottom-up PBPK model is built using in vitro solubility and dissolution data for predicting food effect. For a poorly soluble weak base, PBPK models are likely to predict an absorption limited by precipitation. The extent of supersaturation and precipitation depends on the difference between gastric and intestinal $\mathrm{pH}$. Since food affects the gastric $\mathrm{pH}$, the exposure of a poorly soluble weak base is expected to show a food effect. In the absence of established confidence in the mechanism of precipitation (impossible to verify due to non-identifiability), prospective predictions of food and PPI effects based on the PBPK model may lead to resource-intensive studies and futile study designs, costing valuable time and money. An alternative approach may be to first establish confidence in a PBPK model that is meant for food effect prediction. According to Fig. 1, establishing confidence in a PBPK model that is meant for absorption-related application requires an understanding of mechanisms contributing to gut bioavailability and a quantitative assessment of fraction absorbed and gut 


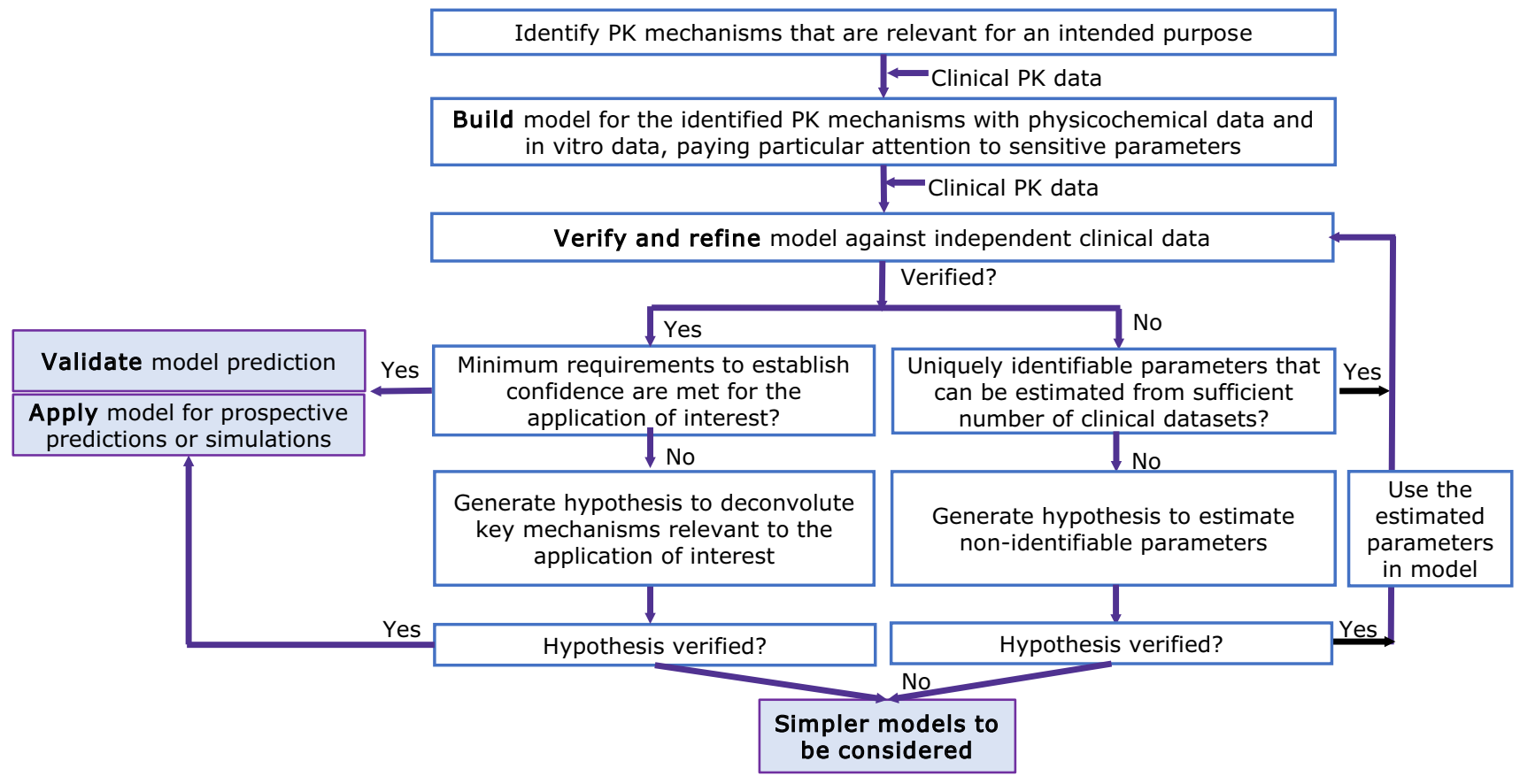

Fig. 4 Workflow to decide between establishing confidence in the application of PBPK model or situations in which simpler models for an intended purpose may be considered. Start with identifying the key PK mechanisms that are relevant for the intended purpose of the application. Next, build the model, ensuring that parameters needed for these mechanisms, especially the sensitive parameters, are estimated from clinical PK data. Verify the model and refine the parameters if necessary. If minimum requirements to establish confidence in

metabolism. The key mechanism that needs to be wellcharacterized from clinical data is intestinal precipitation due to poor solubility. This requires clinical intravenous PK data in addition to oral PK to quantify gut bioavailability $\left(F_{\mathrm{g}}=f_{\text {abs }} \times f_{\mathrm{g}}\right)$. Gut bioavailability is derived from oral bioavailability $(F)$ and fraction escaping hepatic clearance $\left(f_{\mathrm{h}}\right)$ $\left[F=f_{\text {abs }} \times f_{\mathrm{g}} \times f_{\mathrm{h}}\right]$. The model is then verified with oral clinical PK data. Even if the verification criterion is met, there is still no confidence in the $f_{\text {abs }}$ as it is non-identifiable from $f_{\mathrm{g}}$. An hypothesis generation as illustrated in Boxes 5 and 6 can distinguish poor in vivo solubility from gut metabolism as the driver of intestinal loss. If the extent of gut metabolism can be verified in the clinic (by comparing the amount of metabolite formed in intravenous vs. oral routes), then the non-identifiability is resolved and the PBPK model can be used for food effect prediction using the model-generated in vivo solubility. Otherwise, a simpler approach by which confidence in the extent of absorption is based on a platform of evidence from preclinical and clinical data may be adopted. Figure 5 illustrates such a platform of evidence for a drug for which hypothesis testing with preclinical PK the model are not met, simpler models should be preferred. Establishing confidence in sensitive PBPK model parameters for the mechanisms that are identified to be relevant to the intended purpose of a PBPK model application and verifying the model are necessary prior to model application. Hypothesis generation/testing can help resolve parameter non-identifiability through deconvolution of underlying mechanisms, and allows for robust parameterization. $P B P K$ physiologically based pharmacokinetics, $P K$ pharmacokinetics

profiles shows that solubility does not limit exposure. This may be supported by a lack of impact of an enabling formulation on drug exposure, as well as by dose proportionality in toxicological studies in preclinical species. Later in the clinic, dose proportionality or supra-dose proportionality observed in SAD and MAD studies, and a short time to reach $C_{\max }$ in oral PK profiles, can add to the evidence that the exposure of the compound is possibly not limited by solubility. Furthermore, a supra-dose-proportional exposure with higher interindividual variability in $C_{\max }$ compared with AUC is probably indicative of gut metabolism, distinct from solubility limitation. When a PPI study of a compound with omeprazole, a drug known to induce gastroparesis [44], shows lower $C_{\max }$ but the same AUC, it may be deduced that the compound behaves like a BCS Class I drug with no solubility- or permeability-limited absorption. Thus, as a drug progresses through the value chain, it is possible to build a platform of evidence to confirm that the drug does not have solubility/dissolution-limited absorption and therefore may not show a food effect. 


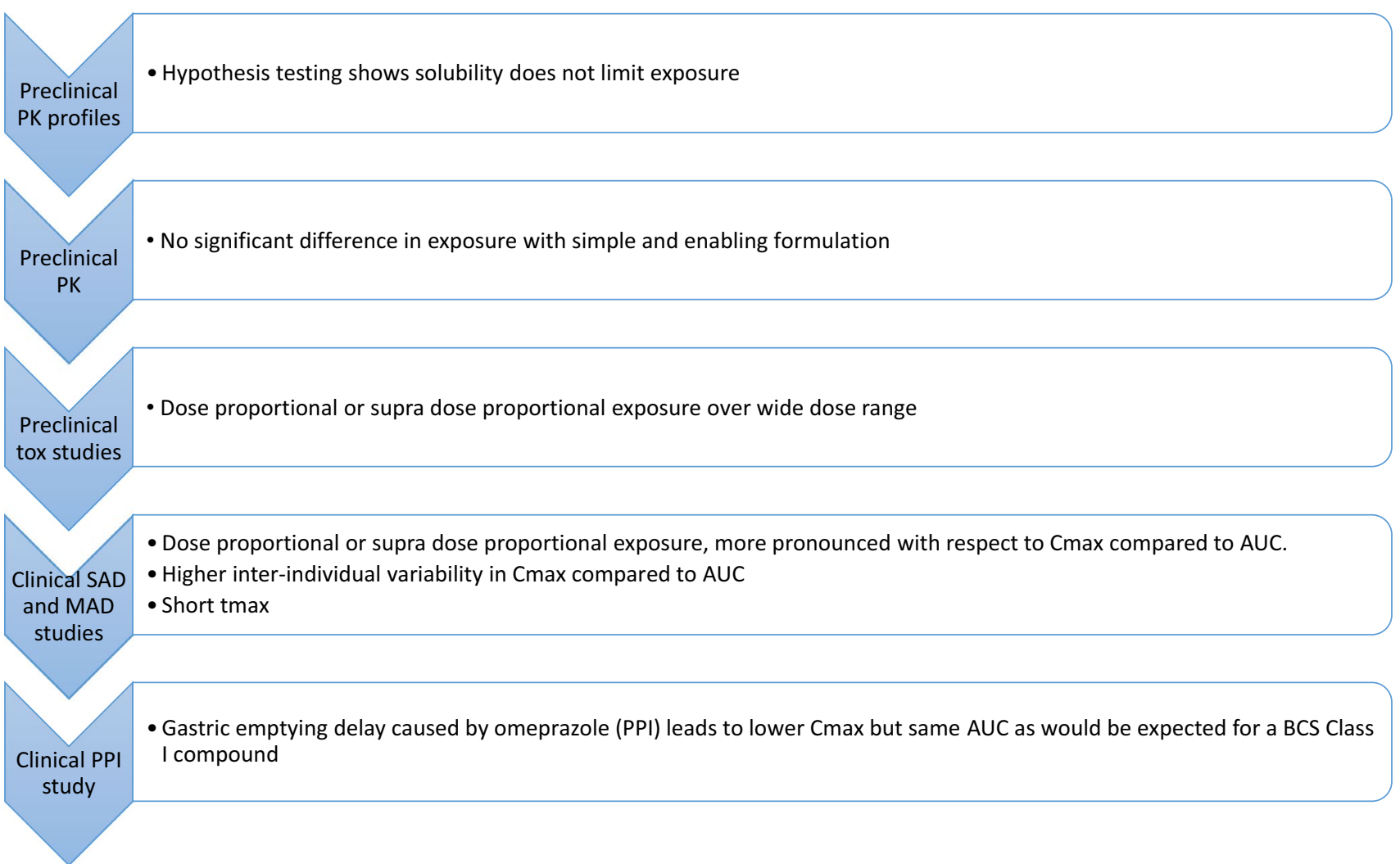

Fig. 5 Building a platform of evidence to enhance confidence in underlying PK mechanisms for a poorly soluble weak base with absorption predicted to be limited by precipitation. PPI proton pump inhibitor, $P K$ pharmacokinetics, tox toxicology, $S A D$ single ascending

\section{Conclusions}

PBPK models are unique in their ability to integrate all available compound and system knowledge for a meaningful prediction of absorption, DDI and drug exposure in an untested scenario or in an unstudied population. Yet, its full potential cannot be unleashed unless confidence in the mechanisms that are relevant to an application are wellestablished. This paper highlights the importance of leveraging all preclinical knowledge to generate hypotheses that can be verified when clinical data become available. If a hypothesis can be verified, then the learning can be incorporated into building a mechanistically credible PBPK model, which is likely to have better predictive performance. In the absence of sufficient clinical datasets to resolve parameter non-identifiability, hypothesis testing offers a great opportunity to maximize the confidence in PBPK model predictions. Continued efforts in improving in vitro assays, gaining a better understanding of the factors driving drug exposure and its variability in different individuals and populations, and improving confidence in system parameters are all imperative to broaden the scope of PBPK applications. As PBPK dose, $M A D$ multiple ascending dose, $C_{\max }$ maximum concentration, $A U C$ area under the curve, $t_{\max }$ time to reach $C_{\max }, B C S$ Biopharmaceutics Classification System

models continue to evolve, building on the collective experience of the scientific community, wider acceptance from regulatory agencies is anticipated in the future.

\section{Compliance with ethical standards}

Conflict of interest Sheila Annie Peters and Hugues Dolgos have no conflicts of interest to declare.

Funding The work reported in this article was not funded/sponsored.

Open Access This article is distributed under the terms of the Creative Commons Attribution-NonCommercial 4.0 International License (http://creativecommons.org/licenses/by-nc/4.0/), which permits any noncommercial use, distribution, and reproduction in any medium, provided you give appropriate credit to the original author(s) and the source, provide a link to the Creative Commons license, and indicate if changes were made.

\section{References}

1. Jones HM, et al. Physiologically based pharmacokinetic modeling in drug discovery and development: a pharmaceutical industry perspective. Clin Pharmacol Ther. 2015;97:247-62. 
2. Peters SA. Physiologically-based pharmacokinetic (PBPK) modelling and simulations: principles, methods and applications in the pharmaceutical industry. Hoboken: Wiley; 2012.

3. Huang SM, Abernethy DR, Wang Y, Zhao P, Zineh I. The utility of modeling and simulation in drug development and regulatory review. J Pharm Sci. 2013;102:2912-23.

4. Zhuang $\mathrm{X}, \mathrm{Lu} \mathrm{C}$. PBPK modeling and simulation in drug research and development. Acta Pharm Sin B. 2016;6:430-40.

5. Luzon E, Blake K, Cole S, Nordmark A, Versantvoort C, Berglund EG. Physiologically based pharmacokinetic modeling in regulatory decision-making at the European Medicines Agency. Clin Pharmacol Ther. 2017;102(1):98-105.

6. Sato M, et al. Quantitative modeling and simulation in PMDA: a Japanese regulatory perspective. CPT Pharmacomet Syst Pharmacol. 2017;6:413-5.

7. Shebley M, Sandhu P, Emami Riedmaier A, Jamei M, Narayanan R, Patel A, et al. Physiologically-based pharmacokinetic model qualification and reporting. Clin Pharmacol Ther. 2018;104:88-110.

8. Peters SA. Evaluation of a generic physiologically based pharmacokinetic model for line shape analysis. Clin Pharmacokinet. 2008;47:261-75.

9. Rowland M, Peck C, Tucker G. Physiologically-based pharmacokinetics in drug development and regulatory science. Annu Rev Pharmacol Toxicol. 2011;51:45-73.

10. Grimstein M, Yang Y, Zhang X, Grillo J, Huang SM, Zineh I, et al. Physiologically-based pharmacokinetic (PBPK) modeling in regulatory science: an update from the US Food and Drug Administration's Office of Clinical Pharmacology. J Pharm Sci. 2019;108(1):21-5.

11. Wang Y, Huang SM. Commentary on fit-for-purpose models for regulatory applications. J Pharm Sci. 2019;108(1):18-20.

12. European Medicines Agency. Guideline on the reporting of physiologically based pharmacokinetic (PBPK) modelling and simulation; 2018. https://www.ema.europa.eu/documents/scien tific-guideline/guideline-reporting-physiologically-based-pharm acokinetic-pbpk-modelling-simulation_en.pdf.

13. Physiologically based pharmacokinetic analyses-format and content guidance for industry; 2018. https://www.fda.gov/regul atory-information/search-fda-guidance-documents/physiologi cally-basedpharmacokinetic-analyses-format-and-content-guida nce-industry.

14. Mehrotra N, Bhattaram A, Earp JC, Florian J, Krudys K, Lee $\mathrm{JE}$, et al. Role of quantitative clinical pharmacology in pediatric approval and labeling. Drug Metab Dispos. 2016;44(7):924-33.

15. European Medicines Agency. European Federation of Pharmaceutical Industries and Associations modelling and simulation workshop report. https://www.ema.europa.eu/en/documents/ report/european-federation-pharmaceutical-inudustries-assoc iationseuropean-medicines-agency-modelling_en.pdf.

16. Manolis E, Rohou S, Hemmings R, Salmonson T, Karlsson M, Milligan PA. The role of modeling and simulation in development and registration of medicinal products: output from the EFPIA/EMA modeling and simulation workshop. CPT Pharmacomet Syst Pharmacol. 2013;2:e31.

17. Abduljalil K, Cain T, Humphries H, Rostami-Hodjegan A. Deciding on success criteria for predictability of pharmacokinetic parameters from in vitro studies: an analysis based on in vivo observations. Drug Metab Dispos. 2014;42:1478-84.

18. Rostami-Hodjegan A. Reverse translation in PBPK and QSP: going backwards in order to go forward with confidence. Clin Pharmacol Ther. 2018;103:224-32.

19. Yau E, Petersson C, Dolgos H, Peters SA. A comparative evaluation of models to predict human intestinal metabolism from nonclinical data. Biopharm Drug Dispos. 2017;38(3):163-86.
20. Johnson TN, Zhou D, Bui KH. Development of physiologically based pharmacokinetic model to evaluate the relative systemic exposure to quetiapine after administration of IR and XR formulations to adults, children and adolescents. Biopharm Drug Dispos. 2014;35:341-52.

21. Hansmann S, Darwich A, Margolskee A, Aarons L, Dressman J. Forecasting oral absorption across biopharmaceutics classification system classes with physiologically based pharmacokinetic models. J Pharm Pharmacol. 2016;68(12):1501-15.

22. Dodd S, Kollipara S, Sanchez-Felix M, Kim H, Meng Q, Beato $S$, et al. Prediction of ARA/PPI drug-drug interactions at the drug discovery and development interface. J Pharm Sci. 2019;108(1):87-101.

23. Samant T, et al. Ribociclib bioavailability is not affected by gastric $\mathrm{pH}$ changes or food intake: in silico and clinical evaluations. Clin Pharmacol Ther. 2018;104(2):374-83.

24. Tistaert C, Heimbach T, Xia B, Parrott N, Samant TS, Kesisoglou F. Food effect projections via physiologically based pharmacokinetic modeling: predictive case studies. J Pharm Sci. 2019;108(1):592-602.

25. Rekic D, Roshammar D, Mukonzo J, Ashton M. In silico prediction of efavirenz and rifampicin drug-drug interaction considering weight and CYP2B6 phenotype. Br J Clin Pharmacol. 2011;71:536-43.

26. Mao J, Johnson TR, Shen Z, Yamazaki S. Prediction of crizotinib-midazolam interaction using the Simcyp population-based simulator: comparison of CYP3A time-dependent inhibition between human liver microsomes versus hepatocytes. Drug Metab Dispos. 2013;41:343-52.

27. Yamazaki S, Johnson TR, Smith BJ. Prediction of drug-drug interactions with crizotinib as the CYP3A substrate using a physiologically based pharmacokinetic model. Drug Metab Dispos. 2015;43:1417-29.

28. Arya V, Zhao P, Reynolds KS, Mishra P, Younis IR. Utilizing PBPK modeling to evaluate the potential of a significant drugdrug interaction between clopidogrel and dasabuvir: a scientific perspective. Clin Pharmacol Ther. 2017;102(4):578-80.

29. Shebley M, Fu W, Badri P, Bow D, Fischer V. Physiologically based pharmacokinetic modeling suggests limited drug-drug interaction between clopidogrel and dasabuvir. Clin Pharmacol Ther. 2017;102(4):679-87.

30. Einolf HJ, et al. Physiologically-based pharmacokinetic model predictions of panobinostat (LBH589) as a victim and perpetrator of drug-drug interactions. Drug Metab Dispos. 2017;45(12):1304-16.

31. Rose RH, Neuhoff S, Abduljalil K, Chetty M, Rostami-Hodjegan A, Jamei M. Application of a physiologically based pharmacokinetic model to predict OATP1B1-related variability in pharmacodynamics of rosuvastatin. CPT Pharmacomet Syst Pharmacol. 2014;3:e124.

32. Snoeys J, Beumont M, Monshouwer M, Ouwerkerk-Mahadevan $S$. Mechanistic understanding of the nonlinear pharmacokinetics and intersubject variability of simeprevir: a PBPK-guided drug development approach. Clin Pharmacol Ther. 2016;99(2):224-34.

33. Shebley M, Liu J, Kavetskaia O, Sydor J, de Morais SM, Fischer $\mathrm{V}$, et al. Mechanisms and predictions of drug-drug interactions of the hepatitis $\mathrm{c}$ virus three direct-acting antiviral regimen: paritaprevir/ritonavir, ombitasvir, and dasabuvir. Drug Metab Dispos. 2017;45(7):755-64.

34. Mao J, Doshi U, Wright M, Hop CECA, Li AP, Chen Y. Prediction of the pharmacokinetics of pravastatin as an OATP substrate using plateable human hepatocytes with human plasma data and PBPK modeling. CPT Pharmacomet Syst Pharmacol. 2018;7(4):251-8.

35. Chen Y, Zhu R, Ma F, Mao J, Chen EC, Choo EF, et al. Assessment of OATP transporter-mediated drug-drug interaction using 
physiologically-based pharmacokinetic (PBPK) modeling — a case example. Biopharm Drug Dispos. 2018;39(9):420-30.

36. Graham L, Lloyd S. Biomedical accelerator mass spectrometry: recent applications in metabolism and pharmacokinetics. Expert Opin Drug Metab Toxicol. 2008;4(8):1021-33.

37. Penner N, Klunk LJ, Prakash C. Human radiolabeled mass balance studies: objectives, utilities and limitations. Biopharm Drug Dispos. 2009;30:185-203.

38. Tsamandouras N, Rostami-Hodjegan A, Aarons L. Combining the 'bottom up' and 'top down' approaches in pharmacokinetic modelling: fitting PBPK models to observed clinical data. $\mathrm{Br} \mathbf{J}$ Clin Pharmacol. 2015;79(1):48-55.

39. Cubitt HE, Yeo KR, Howgate EM, Rostami-Hodjegan A, Barter ZE. Sources of interindividual variability in IVIVE of clearance: an investigation into the prediction of benzodiazepine clearance using a mechanistic population-based pharmacokinetic model. Xenobiotica. 2011;41(8):623-38.
40. McNally K, Cotton R, Loizou GD. Front Pharmacol. A Workflow for Global Sensitivity Analysis of PBPK Models. 2011;23:2-31.

41. Peters SA, Hultin L. Early identification of drug-induced impairment of gastric emptying through physiologically based pharmacokinetic (PBPK) simulation of plasma concentration-time profiles in rat. J Pharmacokinet Pharmacodyn. 2008;35:1-30.

42. Peters SA. Identification of intestinal loss of a drug through physiologically based pharmacokinetic simulation of plasma concentration-time profiles. Clin Pharmacokinet. 2008;47:245-59.

43. EFPIA MID3 Workgroup, Marshall SF, Burghaus R, Cosson V, Cheung SYA, Chenel M, et al. Good practices in model-informed drug discovery and development: practice, application, and documentation. CPT Pharmacomet Syst Pharmacol. 2016;5(3):93-122.

44. Parkman HP, Urbain JL, Knight LC, Brown KL, Trate DM, Miller MA, et al. Effect of gastric acid suppressants on human gastric motility. Gut. 1998;42(2):243-50. 